\title{
Nikotinabhängigkeit
}

\section{Zu geringe Anstrengungen bei der Rauchentwöhnung}

— Viele Risiken des Nikotinkonsums sind bekannt - trotzdem greift jeder vierte Erwachsene in Deutschland täglich zur Zigarette. Doch wer mit dem Rauchen aufhören möchte, erhält hierzulande wenig Unterstützung. Darauf wiesen Experten und Fachgesellschaften auf dem 52. Kongress der DGP in Dresden hin. Sie fordern von der Politik eine Verbesserung der bestehenden Rauchentwöhnungsinfrastruktur und größere Anstrengungen bei der Umsetzung des Nicht-

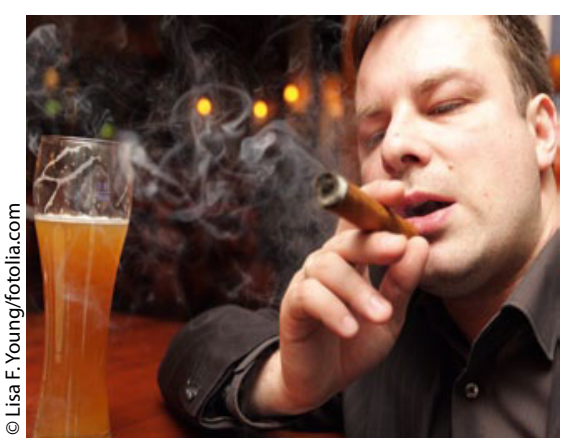

Raucher benötigen mehr Hilfe beim Entzug. raucherschutzes. Zu diesen Ergebnissen kommt auch der aktuelle EQUIPP-Report [1], der erstmals die Fortschritte von 20 europäischen Ländern zum Nichtraucherschutz und zur Bekämpfung der Tabakabhängigkeit analysiert.

\section{Medikamentöse Unterstützung erhöht Abstinenzrate}

"Gerade im Hinblick auf die COPD ist die Tabakentwöhnung nicht nur die wirksamste, sondern auch die kosteneffektivste Maßnahme, um das Voranschreiten zu stoppen und damit, auch kurzfristig, weitere Kosten zu sparen", erklärte Prof. Stefan Andreas, Leiter der Tabakprävention bei der DGP, Immenhausen.„,Dabei hat sich die medikamentöse Unterstützung in Verbindung mit psychosozialer Begleitung für COPD-Patienten als effektiv erwiesen."

Die Wirksamkeit von Vareniclin (Champix ${ }^{\circledR}$ ) zur Rauchentwöhnung belegt eine aktuelle randomisierte, placebokontrollierte Multizenterstudie mit 499 stark abhängigen Rauchern (Fagerström-Tests $\emptyset 6,1$ von 10 Pkt.) mit leich- ter bis mittelgradiger COPD (Alter $\geq 35$ Jahre), die im Jahr vor Beginn der Studie mindestens zehn Zigaretten täglich rauchten [2]. Sie erhielten randomisiert über zwölf Wochen entweder zweimal $1 \mathrm{mg}$ Vareniclin pro Tag $(n=248)$ oder Placebo $(n=251)$ und wurden bis zu einem Jahr weiter beobachtet.

Eine kontinuierliche Rauchabstinenz in den letzten vier Behandlungswochen (Wo. 9-12, prim. Endpunkt) erreichten 42,3\% der Probanden mit Vareniclin gegenüber $8,8 \%$ unter Placebo ( $p<0,0001)$. Auch über die Wochen 9-52 war die kontinuierliche Abstinenzrate unter Vareniclin mit 18,6\% signifikant höher als unter Placebo $(5,6 \%, \mathrm{p}<0,0001)$. Vareniclin wurde in der Untersuchung generell gut vertragen. Die häufigsten Nebenwirkungen waren Übelkeit, abnorme Träume und Schlaflosigkeit.

red

Quellen: 1. Europe Quitting: Progress and Pathways, März 2011, 2. Taschkin D et al. Chest 2010 3. Nach Informationen von Pfizer pharma, Berlin, 2.5.2011

\section{Auch ältere Patienten profitieren}

— Ein Bronchialkarzinom tritt meist bei älteren Patienten auf. Bei ihnen stellt sich die Frage, welche Therapie das Leben verlängern kann, ohne die Lebensqualität zu beeinträchtigen, erklärte Dr. med. Corinna Eschbach, Hamburg. Entscheidend für die Wahl der Therapie sind die Begleiterkrankungen, der Allgemeinzustand und der Patientenwille. „Wir müssen uns gut anschauen, welche Patienten wir auswählen“, betonte Eschbach, „aber das Alter allein ist kein Kriterium für eine bestimmte Therapie."

\section{Vergleichbare Wirksamkeit}

Dass eine platinbasierte Kombinationstherapie auch bei älteren Patienten mit einem fortgeschrittenen nicht kleinzelligen Bronchialkarzinom (NSCLC) sinnvoll ist, dazu liegen gute Daten vor. Eine Subgruppenanalyse der Studie ECOG 1594 zeigt, dass ältere Patienten ebenso von dieser Kombination profitieren wie jüngere. Die Lebensverlängerung war in beiden Altersgruppen vergleichbar, obwohl bei Älteren mehr Toxizitäten auftraten.

\section{Tumorhistologie beachten}

Einen hohen Stellenwert für die Therapiewahl hat die Histologie des Tumors. Betrachtet man die Gesamtgruppe der NSCLC hat die Kombination Pemetrexed (Alimta ${ }^{\circledR}$ ) und Cisplatin eine ähnlich gute Wirksamkeit wie die Standardtherapie mit Gemcitabin und Cisplatin. Unterscheidet man zwischen den histologischen Typen, dann stellt sich heraus, dass Patienten mit einem Nichtplattenepithelkarzinom länger leben, wenn sie eine Kombinationstherapie mit Pemetrexed erhalten.

\section{Überlebenszeit steigt}

Einer Phase III-Studie zufolge stieg das mediane Überleben bei Patienten mit Adenokarzinomen von 10,9 Monaten unter Gemcitabin/Cisplatin auf 12,6 Monate unter der Pemetrexed-Kombination. Und auch bei großzelligen Bronchialkarzinomen ist der Multi-Target-Inhibitor überlegen. Hier betrug der Überlebensvorteil 10,4 gegenüber 6,7 Monaten.

Dass diese Kombination auch gut bei älteren Patienten zum Einsatz kommen kann, bestätigten Subgruppenanalysen. Die Therapie war bei Patienten über 65 Jahren vergleichbar effektiv wie bei jüngeren Patienten. Auch in dieser Altersgruppe nutzte Pemetrexed vor allem Patienten mit Nichtplattenepithelkarzinomen. Das Überleben stieg bei den über 65-jährigen Patienten mit Nichtplattenepithelkarzinomen von 9,8 Monaten unter Gemcitabin/Cisplatin auf 11,3 Monate unter Pemetrexed/Cisplatin.

jn

Symposium „Die Therapie des metastasierten NSCLC-Patienten als interdisziplinäre Herausforderung" anlässlich des 52. DGPKongresses am 8. April 2011 in Dresden (Veranstalter: Lilly Pharma) 